\title{
Blind Adaptive Channel Equalization with Performance Analysis
}

\author{
Shiann-Jeng $\mathrm{Yu}^{1}$ and Fang-Biau Ueng ${ }^{2}$ \\ ${ }^{1}$ National Center for High Performance Computing, No. 21 Nan-Ke 3rd Road, Hsin-Shi, Tainan County 744, Taiwan \\ ${ }^{2}$ Department of Electrical Engineering, National Chung-Hsing University, 250 Kuo-Kuang Road, Taichung 402, Taiwan
}

Received 4 March 2005; Revised 25 August 2005; Accepted 26 September 2005

Recommended for Publication by Christoph Mecklenbräuker

A new adaptive multiple-shift correlation (MSC)-based blind channel equalizer (BCE) for multiple FIR channels is proposed. The performance of the MSC-based BCE under channel order mismatches due to small head and tail channel coefficient is investigated. The performance degradation is a function of the optimal output SINR, the optimal output power, and the control vector. This paper also proposes a simple but effective iterative method to improve the performance. Simulation examples are demonstrated to show the effectiveness of the proposed method and the analyses.

Copyright ( 2006 Hindawi Publishing Corporation. All rights reserved.

\section{INTRODUCTION}

Traditional adaptive equalizers are based on the periodic transmission of a known training data sequence in order to identify or equalize a distorted channel with intersymbol interference (ISI). However, the use of training data sequence may be very costly in some applications. Blind channel equalizers (BCE) without training data available receive much attention in recent years [1-15]. Early blind equalization techniques $[1,2]$ exploited the higher order statistics (HOS) of the output to identify the channels. Unfortunately, the HOS-based BCE requires a large number of data samples and huge computation load which limit their applications in fast changing environments.

To circumvent the shortcomings of the HOS-based approaches, second-order statistics (SOS) was considered in BCE. The SOS-based BCE was developed based on cyclostationary characteristics of the signal. The first SOS-based BCE was derived by Tong et al. [3]. They demonstrated that the SOS is sufficient for blind adaptive equalization by using fractionally sampling or using an array of sensors. Since that, extensive researches were explored in the literature. The well-known approaches are the least-squares, the subspace, and the maximum likelihood $[3,8,9]$. These blind equalizers were termed the two-step methods which estimate multiple channel parameters first and then equalize the channels based on the estimated channel parameters. However, the two-step methods are not optimal because they do not take the channel estimation error into account in the second-step optimization procedure. Recently, direct equalization estimators become more attractive [10-13]. The linear prediction-based equalizer was developed by [13]. Work [12] used the adaptive beamforming technique to develop a constrained optimization method. Multiple-shift correlation (MSC) of the signals can be used in a partially adaptive channel equalizer to achieve fast convergence speed and low computation load. These direct equalizers can be adaptive, leading to much simpler realization for practical implementation.

The SOS-based equalizers have the advantages of fast convergence speed and lower computational complexity compared with the HOS-based approaches. Unfortunately, most of the SOS-based equalizers suffer from the performance degradation caused by the model mismatch. The mismatch may be from inadequate channel order estimation due to limited observation data or the small channel coefficients. Practical multipath channels often have small head and tail terms, selection of appropriate channel order may not be an easy task. As shown in [15] that the blind channel equalization/identification methods should model only the "significant part" of the channel composed of the "large" channel coefficient terms. The "small" head and/or tail terms should be neglected to avoid overmodeling the system and causing degradation of the equalization performance. Work [16] presented a new channel order criterion for blind equalization and [15] investigated the robustness of the LS and SS approaches by using the perturbation theory. 
In this paper, we study the steady-state performance of the MSC-based equalizer. We explore the relationship between the output signal-to-interference plus noise ratio (SINR) and the small head and tail terms of the FIR channels. By applying an orthogonalization approximation to the analyses, the output SINR in terms of the small channel coefficients is derived. A degradation factor defined by the output SINR of the MSC-based equalizer over the optimal value is used to examine the performance degradation of the equalizer. We find that the degradation factor is not only a function of the small channel coefficients, but also a function of the optimal output SINR, the optimal output power, and the control vector. To reduce the degradation caused by the small channel coefficients, this paper proposes a simple iterative method. The analysis of the iterative method is also performed. From the analysis results, we identify that the iterative method indeed improves the equalization performance.

\section{SIGNAL MODEL}

Let us consider an array with $p$ antennas. If the received signal is sampled at the symbol rate, the digitized data of the array can be written by [14],

$$
\mathbf{y}(n)=\sum_{i=1}^{q} \mathbf{h}_{i} s(n-i+1)+\mathbf{z}(n),
$$

where $\mathbf{y}(n)=\left[y_{1}(n) y_{2}(n) \cdots y_{p}(n)\right]^{T},\{s(n)\}$ is the input signal symbol sequence, and $\mathbf{z}(n)=\left[z_{1}(n) z_{2}(n) \cdots z_{p}(n)\right]^{T}$ is the additive white Gaussian noise vector. " $T$ " represents the transpose. $s(n)$ is an independent identically distributed (iid) zero-mean sequence with $\mathrm{E}\left\{s(i) s^{*}(j)\right\}=\delta(i-j)$ and is independent of $z_{i}(n)$. The channel parameters $\left\{\mathbf{h}_{i}=\right.$ $\left.\left[h_{1}(i) h_{2}(i) \cdots h_{p}(i)\right]^{T}, i=1,2, \ldots, q\right\}$ contain all the impulse response of the $p$ FIR channels. The channel order of this multiple FIR channel model of (1) is $q-1$. Define the data vector $\mathbf{Y}_{M}(n)=\left[\mathbf{y}^{T}(n) \mathbf{y}^{T}(n-1) \cdots \mathbf{y}^{T}(n-M+1)\right]^{T}$, $\mathbf{Y}_{M}(n)$ can be expressed as

$$
\mathbf{Y}_{M}(n)=\mathbf{B}_{f}(h) \mathbf{S}_{M}(n)+\mathbf{Z}_{M}(n),
$$

where

$$
\begin{aligned}
\mathbf{B}_{f}(h) & =\left[\begin{array}{cccccc}
\mathbf{h}_{1} & \mathbf{h}_{2} & \cdots & \mathbf{h}_{q} & \cdots & 0 \\
\vdots & \ddots & \ddots & \ddots & \ddots & \vdots \\
0 & \cdots & \mathbf{h}_{1} & \mathbf{h}_{2} & \cdots & \mathbf{h}_{q}
\end{array}\right]_{(p M) x(q+M-1)} \\
& =\left[\mathbf{b}_{f 1}, \mathbf{b}_{f 2}, \ldots, \mathbf{b}_{f(q+M-1)}\right.
\end{aligned}
$$

is a block Toeplitz matrix and is full rank. $\mathbf{Z}_{M}(n)=$ $\left[\mathbf{z}^{T}(n) \mathbf{z}^{T}(n-1) \cdots \mathbf{z}^{T}(n-M+1)\right]^{T}, \quad \mathbf{S}_{M}(n)=$ $[s(n) s(n-1) \cdots s(n-q-M+2)]^{T}$ represents the signal sources corresponding to the columns $\mathbf{b}_{f i}(h)$.

The purpose of the equalizer is to provide an estimate of the signal $s(n-d+1)$ with a possible delay of $d-1$ samples. From beamforming point of view [16], $s(n-d+1)$ can be seen as the desired signal and the other signals $s(n-i)$ with $i \neq d-1$ can be virtually seen as the interferers. Tsatsanis and $\mathrm{Xu}$ [12] noted the analogies of (2) to the beamforming problem statement [16] and developed the COM for direct blind equalizers. They found from (3) that if $M \geq d-1 \geq q$, $\mathbf{b}_{f d}=\left[\begin{array}{llllllllll}0 & \cdots & 0 & \mathbf{h}_{q}^{T} & \mathbf{h}_{q-1}^{T} & \cdots & \mathbf{h}_{1}^{T} & 0 & \cdots & 0\end{array}\right]^{T}$ containing the information of all channel parameters can be used in designing the blind equalizer. The COM algorithm can be derived through an optimization problem with multiple constraints. Consider the optimization problem

$$
\min _{\mathbf{W}_{\mathrm{COM}}} \mathbf{W}_{\mathrm{COM}}^{H} \mathbf{R}_{Y_{M}} \mathbf{W}_{\mathrm{COM}} \quad \text { subject to } C_{d}^{H} \mathbf{W}_{\mathrm{COM}}=\mathbf{h} \text {. }
$$

The weight vector of the COM (constrained optimization method) algorithm is given by [12],

$$
\mathbf{W}_{\mathrm{COM}}=R_{Y_{M}}^{-1} C_{d} \Phi^{-1} \theta
$$

where $\Phi=C_{d}^{H} R_{Y_{M}}^{-1} C_{d}, R_{Y_{M}}=\mathrm{E}\left\{\mathbf{Y}_{M}(n) \mathbf{Y}_{M}^{H}(n)\right\}, \theta$ is the eigenvector of $\Phi$ corresponding to the minimum eigenvalue, and

$$
C_{d}=\left[\begin{array}{c}
\mathbf{0}_{p(d-q) \times p(d-q)} \\
\mathbf{I}_{p q \times p q} \\
\mathbf{0}_{p(M-d) \times p(M-d)}
\end{array}\right]_{(p M) x(p q)} .
$$

From (5), the COM constructs $p M$ adaptive weights to estimate a total of $p q$ channel parameters for resolving one of the $M+q-1$ signals of $\boldsymbol{S}_{M}(n)$. Unfortunately, $p M$ is often much greater than $M+q-1$. For example, let $p=10, q=3$, and $M=9$, the number of adaptive weights for the COM is as high as 90, but the number of all the signal sources of $\mathbf{S}_{M}(n)$ is only 11 . Because the convergence speed and computation load of an adaptive algorithm strongly depend on the dimension of the adaptive weights [17], the COM using such big number of adaptive weights to resolve a signal of $S_{M}(n)$ is not efficient. Another approach called the mutually referenced equalizers (MRE) [18] is based on the following observation. Without consideration of the noise, the equalizers have

$$
\begin{aligned}
\mathbf{V}_{k}^{H} \mathbf{Y}_{M}(n)= & \mathbf{V}_{i}^{H} \mathbf{Y}_{M}(n+i-k) \\
& \text { for } i, k=0,1, \ldots, q+M-1, k>i
\end{aligned}
$$

where the $\mathbf{V}_{k}$ are also defined as $k$-delay equalizers. There exist equalizers to achieve perfect symbol recovery. However, in the presence of noise perfect symbol recovery is impossible by using the criterion of (7). Work [18] successfully developed asymptotic algorithms for all equalization delays.

\section{THE PROPOSED PARTIALLY ADAPTIVE CHANNEL EQUALIZER (PACE)}

Consider a shift correlation matrix defined by $\mathbf{R}_{y}(n, n-k)=$ $E\left\{\mathbf{y}(n) \mathbf{y}^{H}(n-k)\right\}$ and is given by

$$
\begin{aligned}
\mathbf{R}_{y}(n, n-k)=\sum_{i=1}^{q} \sum_{j=1}^{q} \mathrm{E}\left\{s(n-i+1) s^{*}(n-j-k+1)\right\} \\
\times \mathbf{h}_{i} \mathbf{h}_{j}^{H}+\mathrm{E}\left\{\mathbf{z}(n) \mathbf{z}^{H}(n-k)\right\} .
\end{aligned}
$$


Consider the algorithm for direct blind adaptive equalization using partially adaptive weights. Let $\mathrm{Y}(n)$ be a vector containing the first $N$ entries of $\mathbf{Y}_{M}(n)$ and be expressed by $\mathbf{Y}(n)=\left[\mathbf{y}^{T}(n) \mathbf{y}^{T}(n-1) \cdots \mathbf{y}^{T}(n-m+2) y_{1}(n-m+\right.$ 1) $\left.y_{2}(n-m+1) \cdots y_{l}(n-m+1)\right]^{T}$, where $m=\lceil N / p\rceil$ and $l=N-(m-1) p \cdot\lceil\cdot\rceil$ denotes the nearest larger integer. By (2) and (3), $\mathbf{Y}(n)$ can be written by

$$
\mathbf{Y}(n)=\mathbf{B}(h) \mathbf{S}(n)+\mathbf{Z}(n)
$$

where the $N \times(q+m-1)$ matrix $\mathbf{B}(h)$ is written by

$$
\begin{aligned}
\mathbf{B}(h) & =\left[\begin{array}{ccccccc}
\mathbf{h}_{1} & \mathbf{h}_{2} & \cdots & \mathbf{h}_{q} & \cdots & 0 & 0 \\
\vdots & \ddots & \ddots & \ddots & \ddots & \ddots & \vdots \\
\vdots & \cdots & \mathbf{h}_{1} & \mathbf{h}_{2} & \cdots & \mathbf{h}_{q} & 0 \\
0 & \cdots & 0 & \overline{\mathbf{h}}_{1} & \overline{\mathbf{h}}_{2} & \cdots & \overline{\mathbf{h}}_{q}
\end{array}\right] \\
& =\left[\mathbf{b}_{1}, \mathbf{b}_{2}, \ldots, \mathbf{b}_{Q}\right]
\end{aligned}
$$

where $Q=q+m-1$ and $\mathbf{b}_{i}$ is the $i$ th column of $\mathbf{B}(h)$. It is noted that $\mathbf{B}(h)$ is a submatrix of $\mathbf{B}_{f}(h)$ and is therefore full rank [19]. In (10), the vector $\overline{\mathbf{h}}_{i}$ consists of the first $l$ entries of $\mathbf{h}_{i}, \mathbf{S}(n)=[s(n) s(n-1) \cdots s(n-Q+1)]^{T}$, and $\mathbf{Z}(n)=$ $\left[\mathbf{z}^{T}(n) \cdots \mathbf{z}^{T}(n-m+2) z_{1}(n-m+1) z_{2}(n-m+1) \cdots z_{l}(n-\right.$ $m+1)]^{T}$ is a $N \times 1$ noise vector. The adaptive array theory [16] states that a $N$-element array has $N-1$ degrees of freedom to resolve at most $N-1$ signal sources including the desired signal and interference. Therefore, we have to select

$$
N>Q
$$

to resolve one of the signal sources of $\mathbf{S}(n)$. If the direction vector $\mathbf{b}_{d}$ is known, the optimal weight vector corresponding to the desired signal $s(n-d+1)$ is given by [16],

$$
\mathbf{w}_{d}=\mu \mathbf{R}_{Y}^{-1} \mathbf{b}_{d}
$$

where $\mathbf{R}_{Y}(n)=\mathrm{E}\left\{\mathbf{Y}(n) \mathbf{Y}^{H}(n)\right\}=\mathbf{B}(h) \mathbf{B}^{H}(h)+\sigma^{2} \mathbf{I}$ and $\mu$ is a scalar. In this paper, $\mu$ is used to normalize the weight vector. Since the direction vector $\mathbf{b}_{d}$ is probably containing a fraction of all the channel parameters, the equalizer using (12) is called the partially adaptive equalizer. Next, we use the MSC of $\mathbf{Y}(n)$ to find the weight vector $\mathbf{w}_{d}$ directly. Consider that

$$
\begin{aligned}
\mathbf{R}_{Y}(n, n-k)= & \mathbf{B}(h) \mathrm{E}\left\{\mathbf{S}(n) \mathbf{S}^{H}(n-k)\right\} \mathbf{B}^{H}(h) \\
& +\mathrm{E}\left\{\mathbf{Z}(n) \mathbf{Z}^{H}(n-k)\right\} .
\end{aligned}
$$

Thus, if $k=Q-1$ and $k \geq m$, (13) can be reduced to

$$
\mathbf{R}_{Y}(n, n-Q+1)=\mathbf{b}_{Q} \mathbf{b}_{1}^{H}
$$

A simple method for extracting $\mathbf{b}_{Q}$ from (14) is selecting a nonzero vector $\mathbf{u}$, which satisfies $\mathbf{b}_{1}^{H} \mathbf{u} \neq 0$. The direction vector $\mathbf{b}_{Q}$ can be found by

$$
\mathbf{R}_{Y}(n, n-Q+1) \mathbf{u}=\mathbf{b}_{Q}\left(\mathbf{b}_{1}^{H} \mathbf{u}\right) \propto \mathbf{b}_{Q}
$$

Similarly, consider another nonzero vector $\mathbf{v}$ with $\mathbf{b}_{Q}^{H} \mathbf{v} \neq 0$, then

$$
\mathbf{R}_{Y}^{H}(n, n-Q+1) \mathbf{v}=\mathbf{b}_{1}\left(\mathbf{b}_{Q}^{H} \mathbf{v}\right) \propto \mathbf{b}_{1} .
$$

By (12), (15), and (16), the weight vectors of $\mathbf{w}_{1}$ and $\mathbf{w}_{Q}$ can be given by

$$
\begin{gathered}
\mathbf{w}_{Q}=\mu \mathbf{R}_{Y}^{-1}(n) \mathbf{R}_{Y}(n, n-Q+1) \mathbf{u} \propto \mathbf{R}_{Y}^{-1}(n) \mathbf{b}_{Q}, \\
\mathbf{w}_{1}=\mu \mathbf{R}_{Y}^{-1}(n) \mathbf{R}_{Y}^{H}(n, n-Q+1) \mathbf{v} \propto \mathbf{R}_{Y}^{-1}(n) \mathbf{b}_{1} .
\end{gathered}
$$

The outputs corresponding to the zero-delay and $(Q-1)$ delay signals are given by $\widehat{s}(n)=\mathbf{w}_{1}^{H}(n) \mathbf{Y}(n)$ and $\hat{s}(n-Q+$ $1)=\mathbf{w}_{Q}^{H}(n) \mathbf{Y}(n)$, respectively. Using the same approach, the weight vectors of $\mathbf{w}_{d}$ for $d=2,3, \ldots,\lceil Q / 2\rceil$ can be derived as follows:

$$
\begin{gathered}
\mathbf{w}_{d}=\mu \mathbf{R}_{Y}^{-1}(n) \mathbf{R}_{Y}(n, n-Q+d) \mathbf{w}_{Q}, \\
\mathbf{w}_{Q+1-d}=\mu \mathbf{R}_{Y}^{-1}(n) \mathbf{R}_{Y}^{H}(n, n-Q+d) \mathbf{w}_{1},
\end{gathered}
$$

where $\mathbf{w}_{Q}=\mu \mathbf{R}_{Y}^{-1}(n) \mathbf{R}_{Y}(n, n-Q+1) \mathbf{u}$ and $\mathbf{w}_{1}=\mu \mathbf{R}_{Y}^{-1}(n) \mathbf{R}_{Y}^{H}(n$, $n-Q+1) \mathbf{v}$. It is noted that the above algorithm needs two initial vectors $\mathbf{u}$ and $\mathbf{v}$ in (17) for calculating $\mathbf{w}_{1}$ and $\mathbf{w}_{Q}$, respectively. In theory, any nonzero vectors having $\mathbf{b}_{Q}^{H} \mathbf{u} \neq 0$ and $\mathbf{b}_{1}^{H} \mathbf{v} \neq 0$ can be chosen as the candidates. We can select $\mathbf{u}=\mathbf{w}_{Q}$ and $\mathbf{v}=\mathbf{w}_{1}$ for consistency of the algorithm. In the next section, we study the equalization performance in the presence of channels with small head and tail channel coefficients. We find that for the batch processing, selecting $\mathbf{u}=\mathbf{w}_{Q}$ and $\mathbf{v}=\mathbf{w}_{1}$ has the benefit of improving the performance. On the consideration of adaptive implementation of the proposed PACE algorithm, we first insert the time index for the weight vectors for clarification. A straightforward thinking is to express (18) as follows:

$$
\begin{gathered}
\mathbf{w}_{d}(n)=\mu \mathbf{R}_{Y}^{-1}(n) \mathbf{R}_{Y}(n, n-Q+d) \mathbf{w}_{Q}(n), \\
\mathbf{w}_{Q+1-d}(n)=\mu \mathbf{R}_{Y}^{-1}(n) \mathbf{R}_{Y}^{H}(n, n-Q+d) \mathbf{w}_{1}(n) .
\end{gathered}
$$

Here, the algorithm cannot be implemented due to unavailability of $\mathbf{w}_{Q}(n)$ at this moment. For the recursive implementation of the PACE, we slightly modify the above equations as

$$
\begin{gathered}
\mathbf{w}_{d}(n)=\mu \mathbf{R}_{Y}^{-1}(n) \mathbf{R}_{Y}(n, n-Q+d) \mathbf{w}_{Q}(n-1), \\
\mathbf{w}_{Q+1-d}(n)=\mu \mathbf{R}_{Y}^{-1}(n) \mathbf{R}_{Y}^{H}(n, n-Q+d) \mathbf{w}_{1}(n-1) .
\end{gathered}
$$

Let the correlation matrix be updated by

$$
\begin{aligned}
\mathbf{R}_{Y}(n, n-k)= & (1-\alpha) \mathbf{R}_{Y}(n-1, n-1-k) \\
& +\alpha \mathbf{Y}(n) \mathbf{Y}^{H}(n-k),
\end{aligned}
$$


where $\alpha$ is a weighting factor with $0 \leq \alpha \leq 1$. The RLS-based PACE algorithm is summarized as follows:

$$
\begin{aligned}
& \mathbf{R}_{Y}^{-1}(n)=\frac{1}{(1-\alpha)} \mathbf{R}_{Y}^{-1}(n-1) \\
&-\frac{\alpha}{(1-\alpha)} \frac{\mathbf{R}_{Y}^{-1}(n-1) \mathbf{Y}(n) \mathbf{Y}^{H}(n) \mathbf{R}_{Y}^{-1}(n-1)}{(1-\alpha)+\alpha \mathbf{Y}^{H}(n) \mathbf{R}_{Y}^{-1}(n-1) \mathbf{Y}(n)}, \\
& \mathbf{R}_{Y}(n, n-Q+d)=(1-\alpha) \mathbf{R}_{Y}(n-1, n-1-Q+d) \\
&+\alpha \mathbf{Y}(n) \mathbf{Y}^{H}(n-Q+d), \\
& \mathbf{P}_{d}(n)= \mathbf{R}_{Y}(n, n-Q+d) \mathbf{w}_{Q}(n-1), \\
& \mathbf{P}_{Q+1-d}(n)= \mathbf{R}_{Y}^{H}(n, n-Q+d) \mathbf{w}_{1}(n-1), \\
& \mathbf{w}_{d}(n)=\widehat{\mathbf{W}}_{d}(n) /\left\|\widehat{\mathbf{W}}_{d}(n)\right\| \\
& \quad \text { with } \widehat{\mathbf{W}}_{d}(n)=\mathbf{R}_{Y}^{-1}(n) \mathbf{P}_{d}(n), \\
& \mathbf{w}_{Q+1-d}(n)=\widehat{\mathbf{W}}_{Q+1-d}(n) /\left\|\widehat{\mathbf{W}}_{Q+1-d}(n)\right\| \\
& \text { with } \widehat{\mathbf{W}}_{Q+1-d}(n)=\mathbf{R}_{Y}^{-1}(n) \mathbf{P}_{Q+1-d}(n),
\end{aligned}
$$

with $\mathbf{R}_{Y}^{-1}(0)=\tau \mathbf{I}$ and $\mathbf{w}_{Q}(0)=\mathbf{u}$ and $\mathbf{w}_{1}(0)=\mathbf{v}$. Here, $\tau$ is a very large scalar. The computational complexity is $\mathbf{O}\left(N^{2}\right)$.

\subsection{A new order detection criterion}

Now consider that

$$
\digamma_{k}=\operatorname{trace}\left\{\mathbf{R}_{Y}^{H}(n, n-k) \mathbf{R}_{Y}^{-1} \mathbf{R}_{Y}(n, n-k)\right\},
$$

we have

$$
\digamma_{k}= \begin{cases}\left\|\mathbf{h}_{q}\right\|^{2}\left(\mathbf{h}_{1}^{H} \mathbf{R}_{Y}^{-1} \mathbf{h}_{1}\right), & \text { if } k=q-1, \\ 0, & \text { if } k \geq q,\end{cases}
$$

where $\left\|\mathbf{h}_{i}\right\|$ denotes the 2-norm of $\mathbf{h}_{i}$. Since $\left\|\mathbf{h}_{i}\right\|$ is not zero, $\digamma_{k}$ may be an indicator for determining the order of the FIR channels by checking its value nonzero. However, at practical situation of finite number of samples, we have

$$
\hat{\digamma}_{k}=\operatorname{trace}\left\{\hat{\mathbf{R}}_{Y}^{H}(n, n-k) \hat{\mathbf{R}}_{Y}^{-1} \widehat{\mathbf{R}}_{Y}(n, n-k)\right\}=\sum_{i=1}^{p} \widehat{g}_{k}(i),
$$

where $\widehat{g}_{k}(i)=\hat{\mathbf{P}}_{i}^{H} \hat{\mathbf{R}}_{Y}^{-1} \widehat{\mathbf{P}}_{i}$ with $\hat{\mathbf{P}}_{i}$ the $i$ th column of $\widehat{\mathbf{R}}_{Y}(n, n-$ $k)$. In practice, $\hat{F}_{k}$ will never be zero for any $k$. Therefore detecting the channel order by nonzero check criteria should be modified for the finite-sample examples.

Here, we observe that the values of $\hat{\digamma}_{k}$ for $k \geq q$ should not have very significant difference at sufficient large number of samples. We suppose that $\hat{\digamma}_{k}$ for $k \geq q$ are in the same hypothesis termed $\mathscr{H}_{0}$. On the other hand, $\hat{\digamma}_{q-1}$ should be in another hypothesis termed $\mathscr{H}_{1}$. Now consider the following parameter:

$$
\Upsilon_{k}=\frac{\hat{\digamma}_{k}}{\sqrt{(1 /(K-k)) \sum_{i=k+1}^{K} \hat{\digamma}_{i}^{2}}},
$$

TABLE 1: Channel impulse response of 4-element array.

\begin{tabular}{c|cccc}
\hline Antenna & $\mathbf{h}_{0} * 10^{3}$ & $\mathbf{h}_{1} * 10^{3}$ & $\mathbf{h}_{2}$ & $\mathbf{h}_{3}$ \\
\hline$\# 1$ & $4.091 \mathrm{e}^{j(-0.019)}$ & $9.06 \mathrm{e}^{j(-0.41)}$ & 0 & $1.31 \mathrm{e}^{j(0.23)}$ \\
$\# 2$ & $2.47 \mathrm{e}^{j(0.58)}$ & $18.4 \mathrm{e}^{j(-1.25)}$ & $1.31 \mathrm{e}^{j(-0.23)}$ & $1.16 \mathrm{e}^{j(1.48)}$ \\
$\# 3$ & $2.74 \mathrm{e}^{j(-0.91)}$ & $6.9 \mathrm{e}^{j(0.92)}$ & 0 & $0.62 \mathrm{e}^{j(-1.11)}$ \\
$\# 4$ & $1.39 \mathrm{e}^{j(-0.03)}$ & $18.4 \mathrm{e}^{j(-1.46)}$ & $0.52 \mathrm{e}^{j(-1.13)}$ & $0.21 \mathrm{e}^{j(-1.43)}$ \\
\hline Antenna & $\mathbf{h}_{4}$ & $\mathbf{h}_{5}$ & $\mathbf{h}_{6} * 10^{3}$ & $\mathbf{h}_{7} * 10^{3}$ \\
\hline$\# 1$ & $2.87 \mathrm{e}^{j(0.98)}$ & $0.32 \mathrm{e}^{j(0.96)}$ & $5.77 \mathrm{e}^{j(-1.16)}$ & $2.56 \mathrm{e}^{j(0.31)}$ \\
$\# 2$ & $2.09 \mathrm{e}^{j(1.01)}$ & $0.75 \mathrm{e}^{j(0.68)}$ & $3.95 \mathrm{e}^{j(0.019)}$ & $1.35 \mathrm{e}^{j(1.28)}$ \\
$\# 3$ & $1.21 \mathrm{e}^{j(-1.08)}$ & $0.15 \mathrm{e}^{j(-0.98)}$ & $13.08 \mathrm{e}^{j(-0.98)}$ & $1.54 \mathrm{e}^{j(-0.74)}$ \\
$\# 4$ & $0.95 \mathrm{e}^{j(-1.07)}$ & $0.31 \mathrm{e}^{j(-0.95)}$ & $15.06 \mathrm{e}^{j(0.88)}$ & $0.37 \mathrm{e}^{j(-1.28)}$ \\
\hline
\end{tabular}

where $K$ is chosen as a sufficient large integer so that $K>q$. Since $\hat{\digamma}_{k}$, for $K \geq k \geq q$, do not have significant difference, the denominator and the nominator of (26) should be approximately equal. It follows that $\Upsilon_{k}$ should be around 1 for $K \geq k \geq q$. On the contrary, since $\hat{\digamma}_{q-1}$ should be significantly greater than $\hat{\digamma}_{k}$ for $K \geq k \geq q, \Upsilon_{q-1}$ should be a significant large value comparing to $\Upsilon_{k}$ for $k \geq q$. Therefore, we propose a detection criterion by

$$
\Upsilon_{k} \begin{cases}\geq \eta, & \text { for } \hat{\digamma}_{k} \text { in } \mathscr{H}_{1}, \\ <\eta, & \text { for } \hat{\digamma}_{k} \text { in } \mathscr{H}_{0}\end{cases}
$$

where $\eta$ is a detection threshold. The channel order $q$ can be determined by $q=k+1$ if $\Upsilon_{k} \geq \eta$. As a fact, large $K$ is preferred, but large $K$ leads to more computations for finding all $\Upsilon_{k}$ for order detection.

It is known that $\hat{\mathbf{R}}_{Y}(n, n-k)$ is the maximum likelihood estimate of $\mathbf{R}_{Y}(n, n-k)$ [17]. From the first and second assumptions of this paper, we know that $\{s(n)\}$ is a zero-mean iid random sequence and $\left\{v_{i}(n)\right\}$ is the additive zero-mean white Gaussian noise. Using the central limit theorem [20], each $\widehat{g}_{k}(i)$ can be asymptotically modeled as an independent $\chi^{2}$ random variable for sufficient large $L[21] . \hat{\digamma}_{k}$ is the sum of $\hat{g}_{k}(i)$ and should have the $\chi^{2}$ distribution. According to the probability theory [20], $\Upsilon_{k}^{2}$ has the $F(1, K-k)$ distribution or $\pm \Upsilon_{k}$ has the $t$-distribution with degrees of freedom $K-k$. Since $\hat{\digamma}_{k}$, for $K \geq k \geq q$, are of the hypothesis $\mathscr{H}_{0}$, we have $-\eta< \pm \Upsilon_{k}<\eta$ or equivalently $\Upsilon_{k}<\eta$ at a specified confidence level. The range $(-\eta, \eta)$ is called the confidence interval at a specified confidence level. In general, $90 \%$ or $95 \%$ confidence levels are commonly used. Table 3 presents the threshold with $90 \%$ and $95 \%$ confidence levels, where $\eta$ is a function of $K-k$ and can be written by $\eta=\eta(K-k)$. Since $\hat{\digamma}_{q-1}$ is not of the hypothesis $\mathscr{H}_{0}, \Upsilon_{q-1}$ should violate the rule of $\Upsilon_{q-1}<\eta$. At that time, the order $q$ can be detected. We summarize the proposed order detection procedure as follows.

Step 1. Select the threshold value $\eta$ based on a specified confidence level and select a sufficient large integer $K$.

Step 2. Compute $\hat{\digamma}_{k}$ by (25) for $k=K, K-1, \ldots, 1$.

Step 3. Starting from $k=K-1$, calculate $\Upsilon_{k}$ by (26). 
TABLE 2: Channel impulse response of 6-element array.

\begin{tabular}{c|llll}
\hline Antenna & \multicolumn{1}{|c}{$\mathbf{h}_{1}$} & \multicolumn{1}{c}{$\mathbf{h}_{2}$} & \multicolumn{1}{c}{$\mathbf{h}_{3}$} & \multicolumn{1}{c}{$\mathbf{h}_{4}$} \\
\hline$\# 1$ & $0.02 \mathrm{e}^{j(-0.07)}$ & $0.13 \mathrm{e}^{j(0.45)}$ & $1.29 \mathrm{e}^{j(-0.98)}$ & $0.85 \mathrm{e}^{j(-0.019)}$ \\
$\# 2$ & $2.08 \mathrm{e}^{j(-0.97)}$ & $1.21 \mathrm{e}^{j(0.78)}$ & $1.31 \mathrm{e}^{j(-1.28)}$ & $0.85 \mathrm{e}^{j(1.11)}$ \\
$\# 3$ & $0.06 \mathrm{e}^{j(-0.02)}$ & $0.45 \mathrm{e}^{j(-0.52)}$ & $0.43 \mathrm{e}^{j(-0.99)}$ & $0.28 \mathrm{e}^{j(-0.019)}$ \\
$\# 4$ & $0.02 \mathrm{e}^{j(-0.19)}$ & $1.091 \mathrm{e}^{j(-0.33)}$ & $0.29 \mathrm{e}^{j(-1.019)}$ & $0.19 \mathrm{e}^{j(0.89)}$ \\
$\# 5$ & $0.15 \mathrm{e}^{j(0.79)}$ & $0.25 \mathrm{e}^{j(-0.79)}$ & $0.24 \mathrm{e}^{j(-0.78)}$ & $0.18 \mathrm{e}^{j(-0.19)}$ \\
$\# 6$ & $0.008 \mathrm{e}^{j(-0.02)}$ & $0.05 \mathrm{e}^{j(-0.55)}$ & $0.09 \mathrm{e}^{j(-1.019)}$ & $0.09 \mathrm{e}^{j(-0.29)}$ \\
\hline
\end{tabular}

Step 4. If $\Upsilon_{k}<\eta, k=k-1$ and go back to Step 3.

Step 5. If $\Upsilon_{k} \geq \eta, q=k+1$ and stop the procedure.

In general, at least a signal source of $\mathbf{S}(n)$ will be resolved in equalization. The minimum criterion for the PACE to equalize the channels and resolve at least two signal sources is $q+m-2 \geq m$, that is, $q \geq 2$. It shows that if at least a multipath signal is present in the environment, the PACE algorithm using (17) can resolve the zero-delay and $(q-m+2)$ delay signals of $\mathbf{S}(n)$.

More generally, the PACE algorithm can resolve any signal source of $\mathbf{S}(n)$ if the time-shift index $k$ satisfies $k \geq m$. From descriptions of the previous subsection, the minimal multiple-shift index $k$ required for resolving all the signal sources is $k=q+m-1-\lceil(q+m-1) / 2\rceil$. As a result, the constraint for resolving all the signal sources of $\mathbf{S}(n)$ is given by

$$
q+m-1-\left\lceil\frac{q+m-1}{2}\right\rceil \geq m,
$$

where $m=\lceil N / p\rceil$. Both (11) and (28) provide designers constraints of the dimension of the partially adaptive weights $N$ to achieve channel equalization.

\section{STEADY-STATE PERFORMANCE ANALYSIS}

For the batch processing, the initial vectors $\mathbf{u}$ and $\mathbf{v}$ which satisfy with the constraints shown in the above section can achieve the same performance. However, in the presence of channel with small head and tail channel coefficients [22], the performance is different. In this section, we study the effect of the initial vectors $\mathbf{u}$ and $\mathbf{v}$ in the steady-state with small channel coefficients. A method for selecting the initial vectors is proposed and its performance is also studied. Let us consider a performance index called the output SINR which is defined as follows:

$$
\xi_{d}=\frac{\mathbf{w}_{d}^{H} \mathbf{b}_{d} \mathbf{b}_{d}^{H} \mathbf{w}_{d}}{\mathbf{w}_{d}^{H}\left(\mathbf{R}_{Y}-\mathbf{b}_{d} \mathbf{b}_{d}^{H}\right) \mathbf{w}_{d}}
$$

for estimating the $(d-1)$-delay signal source of $\mathbf{Y}(n)$, that is, $s(n-d+1)$, by using $\mathbf{w}_{d}$, where $\mathbf{w}_{d}^{H} \mathbf{b}_{d} \mathbf{b}_{d}^{H} \mathbf{w}_{d}$ is called the output signal power corresponding to $s(n-d+1)$.

\subsection{Analysis}

It has been assumed that the direction vectors of $\mathbf{b}_{1}, \mathbf{b}_{2}, \ldots$, $\mathbf{b}_{m_{1}}$ and $\mathbf{b}_{Q}, \mathbf{b}_{Q-1}, \ldots, \mathbf{b}_{Q-m_{2}+1}$ are small comparing with the direction vectors of $\mathbf{b}_{m_{1}+1}, \mathbf{b}_{m_{1}+2}, \ldots, \mathbf{b}_{Q-m_{2}}$. The weight vectors are

$$
\mathbf{w}_{m_{1}+1}=\mathbf{R}_{Y}^{-1}(n) \mathbf{R}_{Y}^{H}\left(Q-m_{1}-m_{2}-1\right) \mathbf{u}
$$

where

$$
\mathbf{R}_{Y}\left(Q-m_{1}-m_{2}-1\right)=\sum_{i=1}^{m_{1}+m_{2}+1} \mathbf{b}_{Q-m_{1}-m_{2}-1+i} \mathbf{b}_{i}^{H} .
$$

Substituting (31) into (30) yields

$$
\mathbf{w}_{m_{1}+1}=\sum_{i=1}^{m_{1}+m_{2}+1} \mathbf{R}_{Y}^{-1}(n) \mathbf{b}_{i} \mathbf{b}_{Q-m_{1}-m_{2}-1+i}^{H} \mathbf{u} .
$$

Using (32), we have

$$
\begin{aligned}
\mathbf{b}_{m_{1}+1}^{H} \mathbf{w}_{m_{1}+1} & =\sum_{i=1}^{m_{1}+m_{2}+1} \mathbf{b}_{m_{1}+1}^{H} \mathbf{R}_{Y}^{-1}(n) \mathbf{b}_{i} \mathbf{b}_{Q-m_{1}-m_{2}-1+i}^{H} \mathbf{u} \\
& \approx p_{o\left(m_{1}+1\right)} \mathbf{b}_{Q-m_{2}}^{H} \mathbf{u}
\end{aligned}
$$

where $p_{o\left(m_{1}+1\right)}=\mathbf{b}_{m_{1}+1}^{H} \mathbf{R}_{Y}^{-1}(n) \mathbf{b}_{m_{1}+1}$ and $\left|\mathbf{b}_{m_{1}+1}^{H} \mathbf{R}_{Y}^{-1}(n) \mathbf{b}_{i}\right| \ll$ $\mathbf{b}_{m_{1}+1}^{H} \mathbf{R}_{Y}^{-1}(n) \mathbf{b}_{m_{1}+1}$. Therefore, its term can be neglected with comparison to the term with $\mathbf{b}_{m_{1}+1}^{H} \mathbf{R}_{Y}^{-1}(n) \mathbf{b}_{m_{1}+1}$. The output power of using $\mathbf{w}_{m_{1}+1}$ is given by

$$
\begin{aligned}
\mathbf{w}_{m_{1}+1}^{H} & \mathbf{R}_{Y}(n) \mathbf{w}_{m_{1}+1} \\
& =\sum_{i=1, j=1}^{m_{1}+m_{2}+1} \mathbf{u}^{H} \mathbf{b}_{Q-m_{1}-m_{2}-1+i} \mathbf{b}_{i}^{H} \mathbf{R}_{Y}^{-1}(n) \mathbf{b}_{j} \mathbf{b}_{Q-m_{1}-m_{2}-1+j}^{H} \mathbf{u} \\
& \approx \sum_{i=1}^{m_{1}+m_{2}+1} p_{o i}\left|\mathbf{u}^{H} \mathbf{b}_{Q-m_{1}-m_{2}-1+i}\right|^{2}
\end{aligned}
$$

The output SINR of $\mathbf{w}_{m_{1}+1}$ is given by

$$
\xi_{m_{1}+1}=\frac{\mathbf{w}_{m_{1}+1}^{H} \mathbf{b}_{m_{1}+1} \mathbf{b}_{m_{1}+1}^{H} \mathbf{w}_{m_{1}+1}}{\mathbf{w}_{m_{1}+1}^{H}\left(\mathbf{R}_{Y}(n)-\mathbf{b}_{m_{1}+1} \mathbf{b}_{m_{1}+1}^{H}\right) \mathbf{w}_{m_{1}+1}} .
$$

After some calculations, we have $\xi_{m_{1}+1} \approx \xi_{o\left(m_{1}+1\right)} \Psi_{m_{1}+1}$, where $\xi_{o\left(m_{1}+1\right)}$ is the optimal SINR and

$$
\begin{aligned}
\Psi_{m_{1}+1}^{-1}= & 1+\left(\frac{\xi_{o\left(m_{1}+1\right)}}{p_{o\left(m_{1}+1\right)}}\right) \\
& \times\left(\frac{\left(\sum_{i=1, i \neq m_{1}+1}^{m_{1}+m_{2}+1} p_{o i}\left|\mathbf{u}^{H} \mathbf{b}_{Q-m_{1}-m_{2}-1+i}\right|^{2}\right)}{p_{o\left(m_{1}+1\right)}\left|\mathbf{b}^{H} \mathbf{b}_{Q-m_{2}}\right|^{2}}\right)
\end{aligned}
$$

being in terms of the effect of the performance due to small heads and small tails of the channel parameters.

\subsection{Selecting initial vectors}

In this section, we propose a simple iterative method to reduce the sensitivity from the small channel coefficients. This method was also ever used for performance improvement 
TABLE 3: The detection threshold of the $t$-distribution with $90 \%$ and $95 \%$ confidence interval.

\begin{tabular}{c|cccccccccc}
\hline$K-k$ & 1 & 2 & 3 & 4 & 5 & 6 & 7 & 8 & 9 & $\infty$ \\
\hline $90 \%$ & 6.314 & 2.920 & 2.353 & 2.132 & 2.015 & 1.943 & 1.985 & 1.860 & 1.833 & 1.645 \\
$95 \%$ & 12.706 & 3.182 & 2.776 & 2.571 & 2.447 & 2.365 & 2.306 & 2.262 & 2.228 & 1.960 \\
\hline
\end{tabular}

of the adaptive spatial filtering. Let $\mathbf{w}_{m_{1}+1}(l)$ and $\mathbf{w}_{Q-m_{2}}(l)$ represent the weight vectors after $l$ iterations. The iterative method is described as follows:

$$
\begin{aligned}
& \mathbf{w}_{m_{1}+1}(l)=\mathbf{R}_{Y}^{-1}(n) \mathbf{R}_{Y}^{H}\left(Q-m_{1}-m_{2}-1\right) \mathbf{u}(l), \\
& \mathbf{w}_{Q-m_{2}}(l)=\mathbf{R}_{Y}^{-1}(n) \mathbf{R}_{Y}\left(Q-m_{1}-m_{2}-1\right) \mathbf{v}(l) .
\end{aligned}
$$

Let $\mathbf{u}(l)=\mathbf{w}_{Q-m_{2}}(l-1)$ and $\mathbf{v}(l)=\mathbf{w}_{m_{1}+1}(l-1)$. We have

$$
\begin{gathered}
\mathbf{w}_{m_{1}+1}(1)=\mathbf{R}_{Y}^{-1}(n) \mathbf{R}_{Y}^{H}\left(Q-m_{1}-m_{2}-1\right) \mathbf{u}(1) \\
\mathbf{w}_{m_{1}+1}(2)=\boldsymbol{\Phi} \mathbf{v}(1)
\end{gathered}
$$

where

$$
\begin{aligned}
\boldsymbol{\Phi} & =\mathbf{R}_{Y}^{-1}(n) \mathbf{R}_{Y}^{H}\left(Q-m_{1}-m_{2}-1\right) \mathbf{R}_{Y}^{-1}(n) \mathbf{R}_{Y}\left(Q-m_{1}-m_{2}-1\right) \\
& \approx \sum_{i=1}^{m_{1}+m_{2}+1} p_{o\left(Q-m_{1}-m_{2}-1+i\right)} \mathbf{R}_{Y}^{-1}(n) \mathbf{b}_{i} \mathbf{b}_{i}^{H} .
\end{aligned}
$$

By (38), we can find that

$$
\begin{aligned}
\mathbf{w}_{m_{1}+1}(2 l+1) & =\boldsymbol{\Phi}^{l} \mathbf{R}_{Y}^{-1}(n) \mathbf{R}_{Y}^{H}\left(Q-m_{1}-m_{2}-1\right) \mathbf{u} \mathbf{w}_{m_{1}+1} \\
& =\boldsymbol{\Phi}^{l} \mathbf{v}(1)
\end{aligned}
$$

And we can approximate $\Phi^{l}$ by

$$
\Phi^{l} \approx \sum_{i=1}^{m_{1}+m_{2}+1} p_{o\left(Q-m_{1}-m_{2}-1+i\right)}^{l} p_{o i}^{l-1} \mathbf{R}_{Y}^{-1}(n) \mathbf{b}_{i} \mathbf{b}_{i}^{H}
$$

The output SINR after $(2 l+1)$ iterations can be found by

$$
\xi_{m_{1}+1}(2 l+1) \approx \xi_{o\left(m_{1}+1\right)} \Psi_{m_{1}+1}(2 l+1)
$$

where

$$
\begin{aligned}
& \Psi_{m_{1}+1}^{-1}(2 l+1) \\
& =1+\left(\frac{\xi_{o\left(m_{1}+1\right)}}{p_{o\left(m_{1}+1\right)}}\right) \\
& \times\left(\frac{\left(\sum_{i=1, i \neq 1}^{m_{1}+m_{2}+1} p_{o\left(Q-m_{1}-m_{2}-1+i\right)}^{2 l} p_{o i}^{2 l+1}\left|\mathbf{u}^{H} \mathbf{b}_{\left(Q-m_{1}-m_{2}-1+i\right)}\right|^{2}\right)}{p_{o\left(Q-m_{2}\right)}^{2 l} p_{o\left(m_{1}+1\right)}^{2 l+1}\left|\mathbf{u}^{H} \mathbf{b}_{Q-m_{2}}\right|^{2}}\right) .
\end{aligned}
$$

\section{SIMULATION RESULTS}

In this section, computer simulations are performed to evaluate the proposed blind equalizer. The COM and MRE are also performed for comparison.

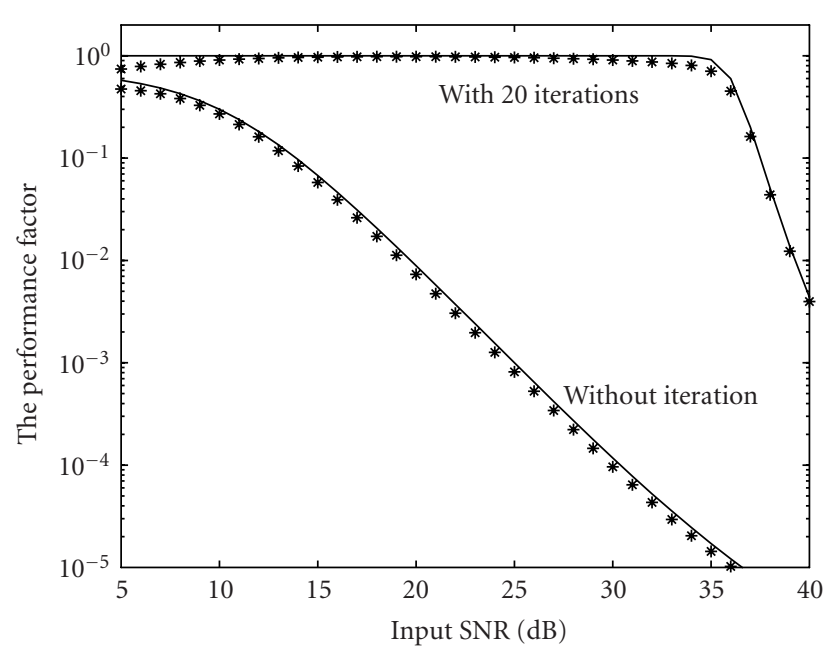

FIGURE 1: The performance factor versus the input SNR with the theoretical results (solid curve) and the experimental results $(*)$.

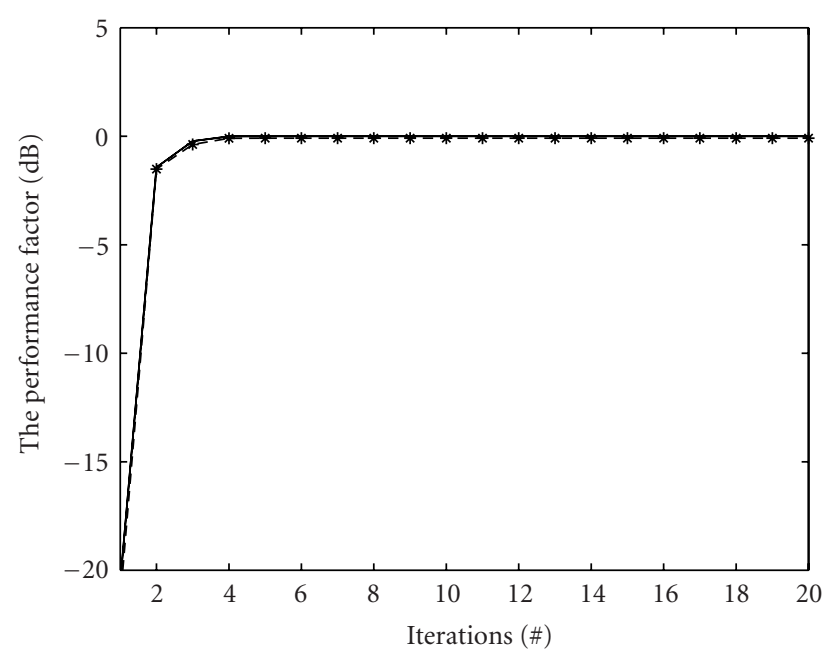

FIGURE 2: The performance factor versus iterations for the batched PACE with the theoretical results (solid curve) and the experimental results $(*)$.

\subsection{Batch processing}

To show the effectiveness of our proposed iterative method, we employ a 4-element array with channel parameters shown in Table 1. It is noted that the channel has 2 small leading and tail channel responses. That is, $m_{1}=m_{2}=2$. The channel order is $7(q=8)$ and the initial vectors are chosen as $\mathbf{u}=\mathbf{v}=[1,1, \ldots, 1]^{T}$. The multiplicity $m$ is chosen 


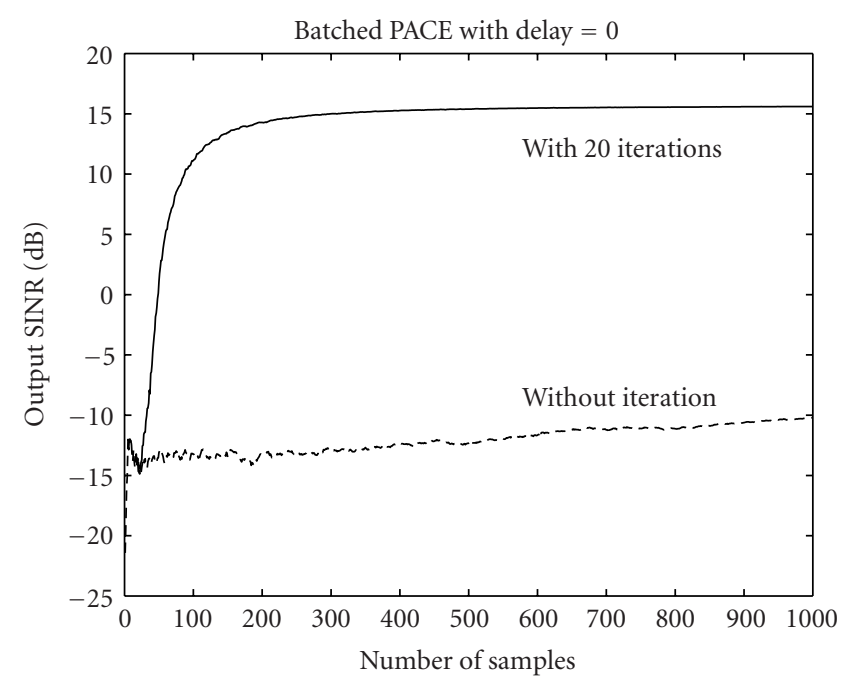

FIGURE 3: The output SINR versus the number of samples for the batched PACE.

as 4 for the proposed algorithm. Thus, we have $Q=11$. Figure 1 shows the performance degradation at different input SNR. It is shown that the iterative method using (37) significantly improves the performance. The PACE without iteration is quite sensitive to the small leading and tail terms. The performance analysis approaches to the experimental result. Figure 2 shows the performance at different number of iterations. The input SNR is $20 \mathrm{~dB}$. We find from these figures that the PACE with iterations can significantly increase the output SINR. However, using iterations more than 15, the improvement is quite limited. Next, let us exam the performance for finite number of samples. The iid BPSK signal with values $\{+1,-1\}$ is passed through the channels and received by the array. The correlation matrix is calculated by

$$
\mathbf{R}_{Y}(n, n-k)=\frac{1}{K} \sum_{i=0}^{K-1} \mathbf{Y}(n-i) \mathbf{Y}^{H}(n-k-i)
$$

with $K$ data samples. Figure 3 shows the results of the PACE with and without iterations. The input SNR is $20 \mathrm{~dB}$. The results are obtained by averaging 100 independent runs. We find that the PACE without iteration does not have good performance. The iterative approach can significantly improve the performance. For the channel order detection problems, we choose $K=8$. The detection thresholds with $90 \%$ and 95\% confidence levels shown in Table 3 are used for simulations. Figure 4 presents the results of the channel order detection of the proposed method with $90 \%$ and $95 \%$ confidence levels and the AIC and MDL. The input signal-tonoise ratio (SNR) is $20 \mathrm{~dB}$. Figure 4 shows that the $\mathrm{AIC}$ and MDL require about 30 samples to detect correct channel order. But the proposed method using both $90 \%$ and $95 \%$ confidence levels requires about 70 and 150 samples, respectively, to achieve the same performance. Figure 5 shows the detection probability versus the number of samples. The results
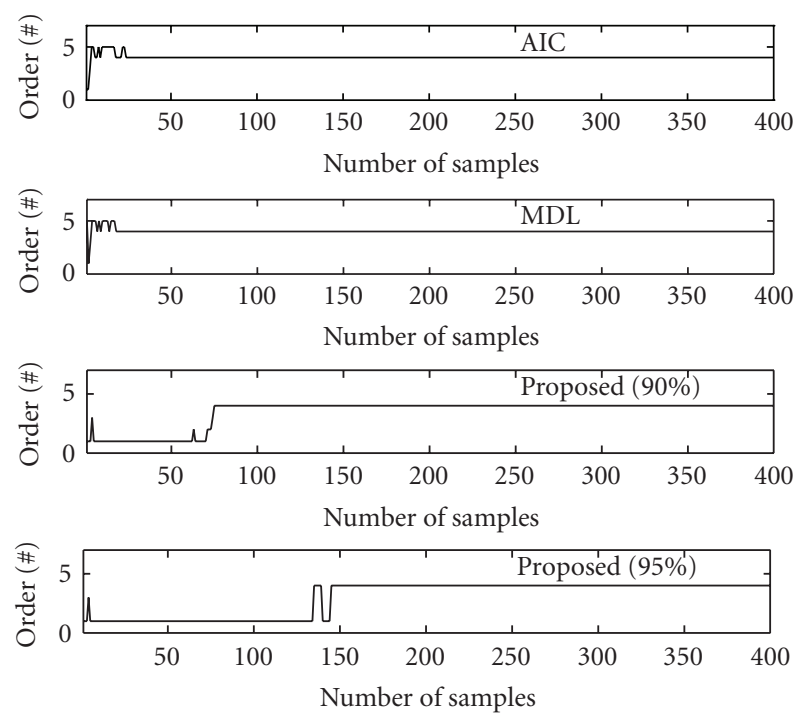

FIgURE 4: Detection of the channel order using the proposed method and the AIC and MDL.

are calculated from 100 independent runs. The input SNR is $20 \mathrm{~dB}$. We find that the MDL is the most efficient method and can detect correct channel order using small number of samples to achieve very high detection probability (over $90 \%$ detection probability). The AIC often overestimates the channel order and its detection probability cannot reach a very high probability. The proposed method using $90 \%$ and $95 \%$ confidence levels works better than the AIC and can achieve very high detection probability if the number of samples is sufficient large. This example shows that using 95\% confidence level is too conservative to detect channel order with high detection probability at limited number of samples. On the contrary, the $90 \%$ confidence level is more moderate for this example. Figure 6 presents the detection probability in different input SNR values. The results are calculated from 100 independent runs. The number of samples used in this example is 200 . We find that the MDL is very sensitive to variations of the input SNR. It cannot reach high detection probability at low input SNR. In contrast, the proposed method using $90 \%$ confidence level is robust to variations of the SNR comparing to the AIC and MDL. At SNR $=5 \mathrm{~dB}$, it can detect correct channel order with more than $70 \%$ probability. In this figure, the proposed method using 95\% confidence level does not have satisfactory results at low SNR. From Figures 5 and 6, we can conclude that the proposed order detection method (e.g., using the $90 \%$ confidence level) is not sensitive to variations of the input SNR, but is sensitive to the number of samples. In general, the large number of samples is required for the proposed method to detect the channel order with high detection probability.

\subsection{Adaptive processing}

To investigate the PACE algorithm of using (20), we consider an array with $p=6$ antenna elements. A channel impulse response shown in Table 2 is used. The channel order is 3 


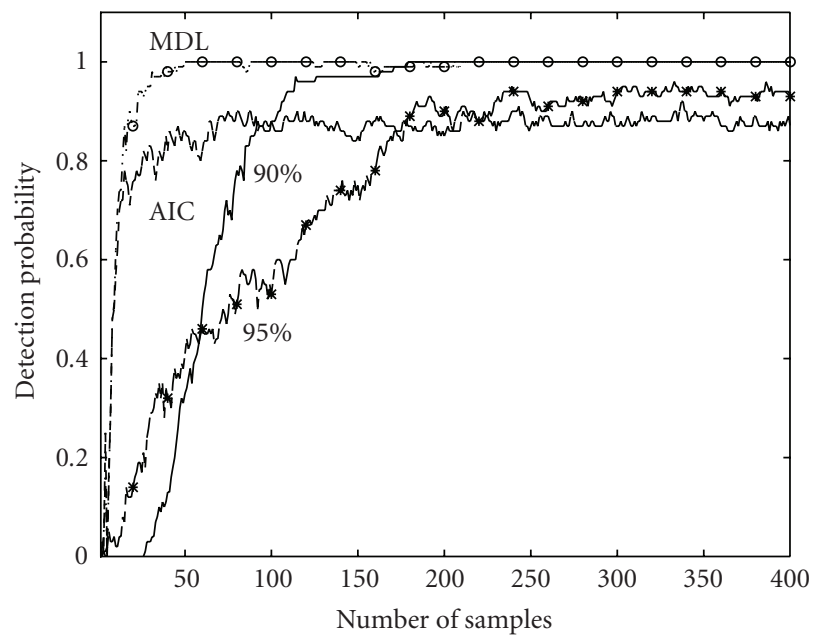

FIgURE 5: The detection probability versus the number of samples.

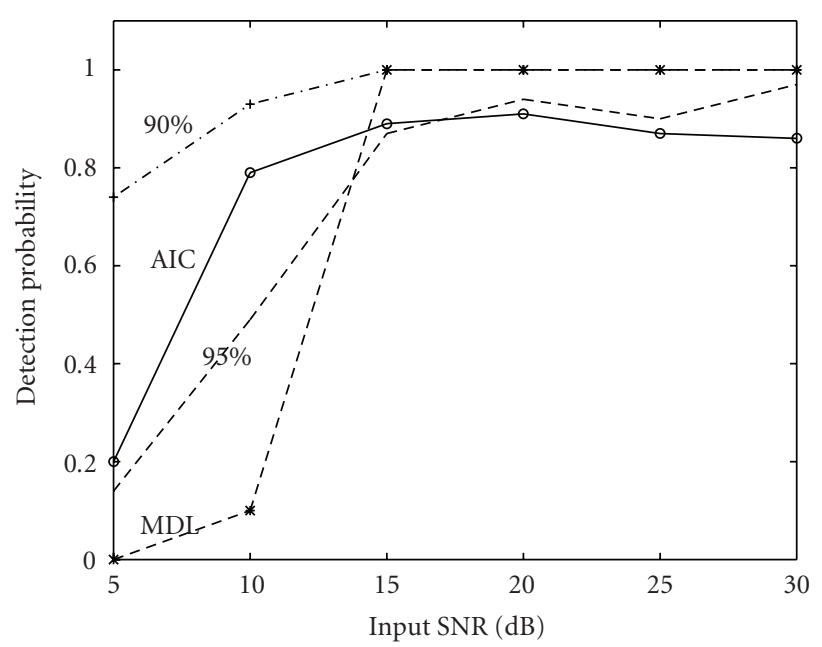

FIGURE 6: The detection probability versus the input SNR.

for this case. The multiplicity $m$ is chosen as 4 for PACE and MRE and as 6 for the COM. At the beginning of the iteration, the initial weight vectors of $\mathbf{w}_{1}(0)$ and $\mathbf{w}_{7}(0)$ of the PACE are set as nonzero random vectors for each independent run. The weighting factor is chosen as $\alpha=1 / n$. Figure 7 presents the output SINR versus the number of samples. The results are obtained by averaging by 100 independent runs. The PACE algorithm has fast convergent speed and achieves very good performance. The PACE has performance better than that of the MRE and COM. We note from (12) that the channel parameters can be estimated from the weight vector. The weight vector $\mathbf{w}_{q} \propto \mathbf{R}_{Y}^{-1} \mathbf{b}_{q}$. Therefore, $\mathbf{b}_{q}=\eta \mathbf{R}_{Y}(n) \mathbf{w}_{q}$, where $\eta$ is a complex variable for gain and phase adjustment. If $m=4$, we have $\mathbf{b}_{q}=\left[\begin{array}{llll}\mathbf{h}_{4}^{T} & \mathbf{h}_{3}^{T} & \mathbf{h}_{2}^{T} & \mathbf{h}_{1}^{T}\end{array}\right]^{T}$. All the channel parameters can be estimated from $\mathbf{b}_{q}$. Figure 8 shows the mean square error (MSE) of the estimated channel parameters. The COM with

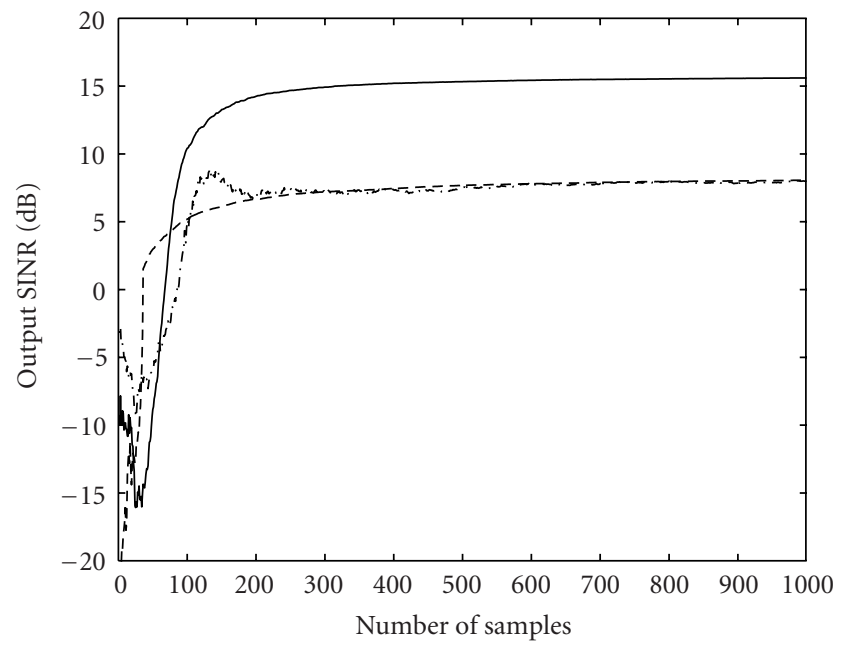

FIgURE 7: The output SINR versus the number of samples. Solid curve: the PACE, dash curve: the COM, dash-dot curve: the MRE.

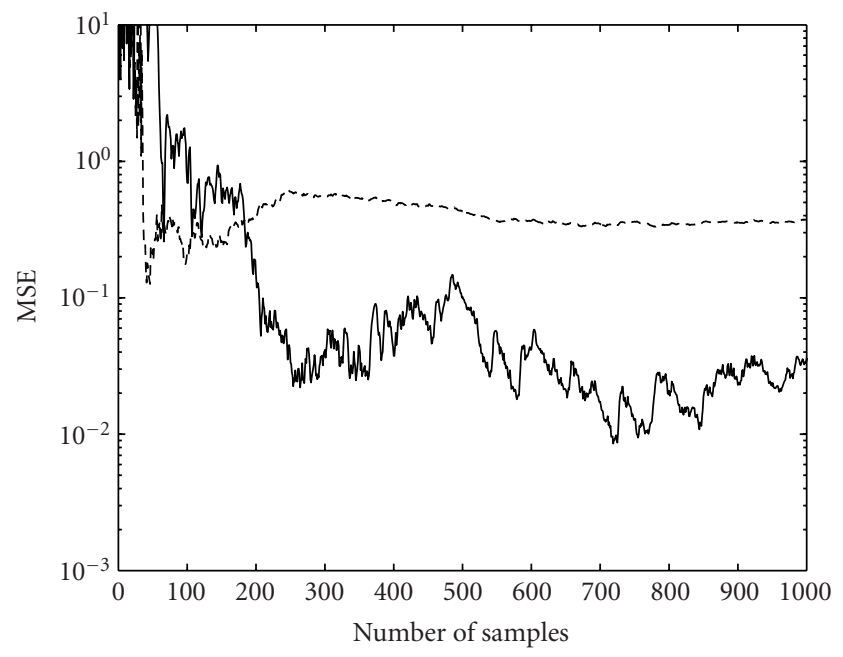

FIGURE 8: The MSE versus the number of samples. Solid curve: the PACE, dash-curve: the COM.

delay 4 and $M=6$ is used for comparison. Figure 8 shows that the PACE outperforms the COM.

\section{CONCLUSIONS}

An effective order detection method and a PACE algorithm for direct multichannel equalization have been presented. Both of the order detection method and the PACE algorithm use the MSC property of the data. The order detection method is derived from the MSC matrix. A $t$-distributionbased hypothesis testing criteria is used for detecting the channel order. The proposed method can effectively detect the channel order without using the EVD or SVD. Simulations show that the method is not sensitive to the input SNR. However, it is sensitive to number of samples. Sufficient large number of samples should be used for the proposed 
detection method to have high detection probability. We have found from the analyses that the weight vector which yields higher output SINR is more sensitive to the small channel coefficients. In order to reduce the performance degradation caused by the small channel coefficients and the control vector, we propose a simple iterative method. The performance improvement of the iterative method has also been analyzed.

\section{REFERENCES}

[1] G. B. Giannakis and J. M. Mendel, "Identification of nonminimum phase systems using higher order statistics," IEEE Transactions on Acoustics, Speech, and Signal Processing, vol. 37, no. 3, pp. 360-377, 1989.

[2] B. Porat and B. Friedlander, "Blind equalization of digital communication channels using high-order moments," IEEE Transactions on Signal Processing, vol. 39, no. 2, pp. 522-526, 1991.

[3] L. Tong, G. Xu, and T. Kailath, "Blind identification and equalization based on second-order statistics: a time domain approach," IEEE Transactions on Information Theory, vol. 40, no. 2, pp. 340-349, 1994.

[4] D. T. M. Slock, "Blind fractionally-spaced equalization, perfect-reconstruction filter banks and multichannel linear prediction," in Proceedings of IEEE International Conference on Acoustics, Speech, and Signal Processing (ICASSP '94), vol. 4, pp. 585-588, Adelaide, SA, Australia, April 1994.

[5] M. Gurelli and C. L. Nikias, "EVAM: an eigenvector-based algorithm for multichannel blind deconvolution of input colored signals," IEEE Transactions on Signal Processing, vol. 43, no. 1, pp. 134-149, 1995.

[6] E. Moulines, P. Duhamel, J.-F. Cardoso, and S. Mayrargue, "Subspace methods for the blind identification of multichannel FIR filters," IEEE Transactions on Signal Processing, vol. 43, no. 2, pp. 516-525, 1995.

[7] H. Liu and G. Xu, "Closed-form blind symbol estimation in digital communications," IEEE Transactions on Signal Processing, vol. 43, no. 11, pp. 2714-2723, 1995.

[8] G. Xu, H. Liu, L. Tong, and T. Kailath, "A least squaresapproach to blind channel identification," IEEE Transactions on Signal Processing, vol. 43, no. 12, pp. 2982-2993, 1995.

[9] Y. Hua, "Fast maximum likelihood for blind identification of multiple FIR channels," IEEE Transactions on Signal Processing, vol. 44, no. 3, pp. 661-672, 1996.

[10] G. B. Giannakis and S. D. Halford, "Blind fractionally spaced equalization of noisy FIR channels: direct and adaptive solutions," IEEE Transactions on Signal Processing, vol. 45, no. 9, pp. 2277-2292, 1997.

[11] G. B. Giannakis and C. Tepedelenlioglu, "Direct blind equalizers of multiple FIR channels: a deterministic approach," IEEE Transactions on Signal Processing, vol. 47, no. 1, pp. 62-74, 1999.

[12] M. K. Tsatsanis and Z. Xu, "Constrained optimization methods for direct blind equalization," IEEE Journal on Selected Areas in Communications, vol. 17, no. 3, pp. 424-433, 1999.

[13] J. Mannerkoski and D. P. Taylor, "Blind equalization using least-squares lattice prediction," IEEE Transactions on Signal Processing, vol. 47, no. 3, pp. 630-640, 1999.

[14] L. T. Tong and Q. Zhao, "Joint order detection and blind channel estimation by least squares smoothing," IEEE Transactions on Signal Processing, vol. 47, no. 9, pp. 2345-2355, 1999.
[15] Q. Zhao and L. T. Tong, "Adaptive blind channel estimation by least squares smoothing," IEEE Transactions on Signal Processing, vol. 47, no. 11, pp. 3000-3012, 1999.

[16] R. T. Compton Jr., Adaptive Antennas, Concepts, and Performance, Prentice-Hall, Englewood Cliffs, NJ, USA, 1988.

[17] R. Monzingo and T. Miller, Introduction to Adaptive Arrays, John Wiley \& Sons, New York, NY, USA, 1980.

[18] D. Gesbert, P. Duhamel, and S. Mayrargue, "On-line blind multichannel equalization based on mutually referenced filters," IEEE Transactions on Signal Processing, vol. 45, no. 9, pp. 2307-2317, 1997.

[19] G. H. Golub and C. F. Van Loan, Matrix Computations, Johns Hopkins University Press, Baltimore, Md, USA, 1983.

[20] P. G. Hoel, S. C. Port, and C. J. Stone, Introduction to Probability Theory, Houghton Mifflin, Boston, Mass, USA, 1971.

[21] A. B. Baggeroer, "Confidence intervals for regression (MEM) spectral estimates," IEEE Transactions on Information Theory, vol. 22 , no. 5, pp. 534-545, 1976.

[22] A. P. Liavas, P. A. Regalia, and J.-P. Delmas, "Blind channel approximation: effective channel order determination," IEEE Transactions on Signal Processing, vol. 47, no. 12, pp. 33363344, 1999.

Shiann-Jeng Yu received the Ph.D. degree from the National Taiwan University in electrical engineering in 1995. From October 1995 to December 2001, he was with National Space Program Office (NSPO) of Taiwan as an Associate Researcher. From January 2001 to July 2002, he was with the National Science Council (NSC) as a Specialist Secretary of vice chairman office. Since August 2002, he has been with the National Center for High Performance Computing (NCHC). He is now a NCHC Deputy Director at the south region office. His specialties and interests are digital signal processing, wireless communication and satellite communication, and grid computing and applications in e-learning.

Fang-Biau Ueng received the Ph.D. degree in electronic engineering from the National Chiao Tung University, Hsinchu, Taiwan in 1995. From 1996 to 2001, he was with National Space Program Office (NSPO) of Taiwan as an Associate Researcher. From 2001 to 2002, he was with Siemens Telecommunication Systems Limited (STSL), Taipei, Taiwan, where he was involved in the design of mobile communication systems. Since

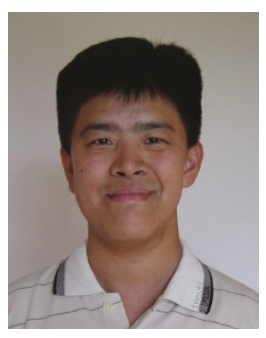
February 2002, he has been with the Department of Electrical Engineering, National Chung-Hsing University, Taichung, Taiwan. His areas of research interests are wireless communication and adaptive signal processing. 